\title{
COINCIDENCE OF ALGEBRAIC AND SMOOTH THETA CORRESPONDENCES
}

\author{
YIXIN BAO AND BINYONG SUN
}

\begin{abstract}
An "automatic continuity" question has naturally occurred since Roger Howe established the local theta correspondence over $\mathbb{R}$ : Does the algebraic version of local theta correspondence over $\mathbb{R}$ agree with the smooth version? We show that the answer is yes, at least when the concerning dual pair has no quaternionic type I irreducible factor.
\end{abstract}

\section{INTRODUCTION}

In his seminal work Ho3, Roger Howe established the smooth version of local theta correspondence over $\mathbb{R}$ as a consequence of its algebraic analogue (see [Ho3, Theorem 1 and Theorem 2.1]). Since then, it has been expected that the smooth version coincides with the algebraic version. Our main goal is to prove this coincidence, at least when the dual pair concerned has no quaternionic type I irreducible factor. In this latter case, we obtain a partial result (cf. Proposition 3.2). The proof is a rather direct application of the conservation relations which are established in $[\mathrm{SZ}]$.

To be precise, let $W$ be a finite-dimensional symplectic space over $\mathbb{R}$ with symplectic form $\langle,\rangle_{W}$. Denote by $\tau$ the involution of $\operatorname{End}_{\mathbb{R}}(W)$ specified by

$$
\langle x \cdot u, v\rangle_{W}=\left\langle u, x^{\tau} \cdot v\right\rangle_{W}, \quad u, v \in W, x \in \operatorname{End}_{\mathbb{R}}(W) .
$$

Let $\left(A, A^{\prime}\right)$ be a pair of $\tau$-stable semisimple real subalgebras of $\operatorname{End}_{\mathbb{R}}(W)$ which are mutual centralizers of each other in $\operatorname{End}_{\mathbb{R}}(W)$. Put

$$
G:=A \bigcap \operatorname{Sp}(W) \text { and } \quad G^{\prime}:=A^{\prime} \bigcap \operatorname{Sp}(W),
$$

which are closed subgroups of the symplectic group $\operatorname{Sp}(W)$. Following Howe, we call the group pair $\left(G, G^{\prime}\right)$ so obtained a reductive dual pair, or a dual pair for simplicity, in $\operatorname{Sp}(W)$.

Write

$$
1 \rightarrow\left\{1, \varepsilon_{W}\right\} \rightarrow \widetilde{\mathrm{Sp}}(W) \rightarrow \mathrm{Sp}(W) \rightarrow 1
$$

for the metaplectic cover of the symplectic group $\operatorname{Sp}(W)$. It does not split unless $W=0$. Denote by $\mathrm{H}(W):=W \times \mathbb{R}$ the Heisenberg group attached to $W$, with the 2017

Received by the editors January 13, 2017, and, in revised form, May 26, 2017, and August 10,

2010 Mathematics Subject Classification. Primary 22E46, 22E50.

Key words and phrases. Reductive dual pair, theta correspondence, Casselman-Wallach representation, Harish-Chandra module.

The second author was supported in part by the National Natural Science Foundation of China (No. 11525105, 11688101, 11621061 and 11531008). 
group multiplication

$$
(u, \alpha)(v, \beta)=\left(u+v, \alpha+\beta+\langle u, v\rangle_{W}\right), \quad u, v \in W, \alpha, \beta \in \mathbb{R} .
$$

Let $\widetilde{\mathrm{Sp}}(W)$ act on $\mathrm{H}(W)$ as group automorphisms through the action of $\operatorname{Sp}(W)$ on $W$. Then we form the semidirect product $\widetilde{\mathrm{J}}(W):=\widetilde{\mathrm{Sp}}(W) \ltimes \mathrm{H}(W)$.

When no confusion is possible, we do not distinguish a representation with its underlying space. Fix an arbitrary non-trivial unitary character $\psi$ on $\mathbb{R}$. Up to isomorphism, there is a unique smooth Fréchet representation (Segal-Shale-Weil representation) $\omega$ of $\widetilde{J}(W)$ of moderate growth such that (see [Sh and We]):

- $\left.\omega\right|_{\mathrm{H}(W)}$ is irreducible and has central character $\psi$;

- $\varepsilon_{W} \in \widetilde{\operatorname{Sp}}(W)$ acts through the scalar multiplication by -1 .

We remark that the second condition is automatic unless $W=0$. The representation $\omega$ may be realized on the space of Schwartz functions on a Lagrangian subspace of $W$ (see Rao).

For every closed subgroup $E$ of $\operatorname{Sp}(W)$, write $\widetilde{E}$ for the double cover of $E$ induced by the metaplectic cover (1.2). Then $\widetilde{G}$ and $\widetilde{G}^{\prime}$ commute with each other inside the group $\widetilde{\operatorname{Sp}}(W)$ (see [MW, Lemma II.5]). Thus the representation $\omega$ induces a representation of $\widetilde{G} \times \widetilde{G}^{\prime}$, which we denote by $\omega_{G, G^{\prime}}$.

For every real reductive group $H$, write $\operatorname{Irr}(H)$ for the set of isomorphism classes of irreducible Casselman-Wallach representations of $H$. Recall that a representation of a real reductive group is called a Casselman-Wallach representation if it is smooth, Fréchet, of moderate growth, and its Harish-Chandra module has finite length. Given a maximal compact subgroup $K_{H}$ of $H$, the Casselman-Wallach globalization theorem asserts that the functor $\mathcal{F}$, which maps a Casselman-Wallach representation of $H$ to its subspace of $K_{H}$-finite vectors, establishes an equivalence between the category of Casselman-Wallach representations of $H$ and the category of finitely generated admissible $\left(\mathfrak{h}, K_{H}\right)$-modules, where $\mathfrak{h}$ denotes the complexified Lie algebra of $H$. The reader is referred to [Ca, Wa, Chapter 11] or [BK for details about Casselman-Wallach representations.

Define

$$
\begin{aligned}
\mathscr{R}^{\infty}\left(\widetilde{G}, \omega_{G, G^{\prime}}\right) & :=\left\{\pi \in \operatorname{Irr}(\widetilde{G}) \mid \operatorname{Hom}_{\widetilde{G}}\left(\omega_{G, G^{\prime}}, \pi\right) \neq 0\right\}, \\
\mathscr{R}^{\infty}\left(\widetilde{G^{\prime}}, \omega_{G, G^{\prime}}\right) & :=\left\{\pi^{\prime} \in \operatorname{Irr}\left(\widetilde{G^{\prime}}\right) \mid \operatorname{Hom}_{\widetilde{G^{\prime}}}\left(\omega_{G, G^{\prime}}, \pi^{\prime}\right) \neq 0\right\}, \\
\mathscr{R}^{\infty}\left(\widetilde{G} \times \widetilde{G^{\prime}}, \omega_{G, G^{\prime}}\right) & :=\left\{\left(\pi, \pi^{\prime}\right) \in \operatorname{Irr}(\widetilde{G}) \times \operatorname{Irr}\left(\widetilde{G^{\prime}}\right) \mid \operatorname{Hom}_{\widetilde{G} \times \widetilde{G^{\prime}}}\left(\omega_{G, G^{\prime}}, \pi \widehat{\otimes} \pi^{\prime}\right) \neq 0\right\} .
\end{aligned}
$$

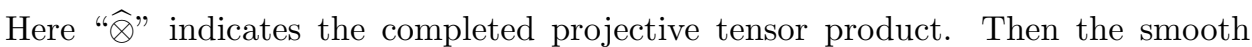
version of the archimedean theta correspondence asserts that $\mathscr{R}^{\infty}\left(\widetilde{G} \times \widetilde{G^{\prime}}, \omega_{G, G^{\prime}}\right)$ is the graph of a bijection (smooth theta correspondence) between $\mathscr{R}^{\infty}\left(\widetilde{G}, \omega_{G, G^{\prime}}\right)$ and $\mathscr{R}^{\infty}\left(\widetilde{G^{\prime}}, \omega_{G, G^{\prime}}\right)$.

We need the following result to have a clean and uniform formulation of the "automatic continuity" problem.

Proposition 1.1. Up to conjugation by $G \times G^{\prime}$, there exists a unique Cartan involution $\theta$ of $\operatorname{Sp}(W)$ such that

$$
\theta(G)=G \quad \text { and } \quad \theta\left(G^{\prime}\right)=G^{\prime} .
$$

The existence part of Proposition 1.1 is well known, by using the classification of reductive dual pairs (see [Ho3, Section 2]). The uniqueness part may also be known 
to experts. But to our best knowledge, it has not been explicitly formulated in the literature. In Section 2, we will sketch a proof for a stronger version of Proposition 1.1 .

Now let $\theta$ be as in Proposition 1.1. Then $\theta$ restricts to Cartan involutions on $G$ and $G^{\prime}$. Put

$$
C:=(\operatorname{Sp}(W))^{\theta}, \quad K:=G^{\theta} \quad \text { and } \quad K^{\prime}:=G^{\prime \theta} \quad \text { (the fixed point groups). }
$$

They are maximal compact subgroups of $\operatorname{Sp}(W), G$ and $G^{\prime}$, respectively.

For every $\pi \in \operatorname{Irr}(\widetilde{G})$, define

$$
\operatorname{Hom}_{\widetilde{G}}^{\mathrm{alg}}\left(\omega_{G, G^{\prime}}, \pi\right):=\operatorname{Hom}_{\mathfrak{g}, \widetilde{K}}\left(\omega_{G, G^{\prime}}^{\mathrm{alg}}, \pi^{\mathrm{alg}}\right),
$$

where $\mathfrak{g}$ denotes the complexified Lie algebra of $G, \omega_{G, G^{\prime}}^{\text {alg }}$ denotes the $\left(\mathfrak{s p}(W) \otimes_{\mathbb{R}}\right.$ $\mathbb{C}, \widetilde{C})$-module of $\widetilde{C}$-finite vectors in $\omega=\omega_{G, G^{\prime}}$, and $\pi^{\text {alg }}$ denotes the $(\mathfrak{g}, \widetilde{K})$-module of $\widetilde{K}$-finite vectors in $\pi$. We define $\operatorname{Hom}_{\widetilde{G^{\prime}}}^{\operatorname{alg}}\left(\omega_{G, G^{\prime}}, \pi^{\prime}\right)$ and $\operatorname{Hom}_{\widetilde{G} \times \widetilde{G^{\prime}}}^{\operatorname{alg}}\left(\omega_{G, G^{\prime}}, \pi \widehat{\otimes} \pi^{\prime}\right)$ in an analogous way, where $\pi^{\prime} \in \operatorname{Irr}\left(\widetilde{G^{\prime}}\right)$. In this algebraic setting, we define

$$
\begin{aligned}
\mathscr{R}^{\operatorname{alg}}\left(\widetilde{G}, \omega_{G, G^{\prime}}\right) & :=\left\{\pi \in \operatorname{Irr}(\widetilde{G}) \mid \operatorname{Hom}_{\widetilde{G}}^{\operatorname{alg}}\left(\omega_{G, G^{\prime}}, \pi\right) \neq 0\right\}, \\
\mathscr{R}^{\operatorname{alg}}\left(\widetilde{G^{\prime}}, \omega_{G, G^{\prime}}\right) & :=\left\{\pi^{\prime} \in \operatorname{Irr}\left(\widetilde{G^{\prime}}\right) \mid \operatorname{Hom}_{\widetilde{G^{\prime}}}^{\text {alg }}\left(\omega_{G, G^{\prime}}, \pi^{\prime}\right) \neq 0\right\}, \\
\mathscr{R}^{\operatorname{alg}}\left(\widetilde{G} \times \widetilde{G^{\prime}}, \omega_{G, G^{\prime}}\right) & :=\left\{\left(\pi, \pi^{\prime}\right) \in \operatorname{Irr}(\widetilde{G}) \times \operatorname{Irr}\left(\widetilde{G^{\prime}}\right) \mid \operatorname{Hom}_{\widetilde{G} \times \widetilde{G^{\prime}}}^{\text {alg }}\left(\omega_{G, G^{\prime}}, \pi \widehat{\otimes} \pi^{\prime}\right) \neq 0\right\} .
\end{aligned}
$$

It is clear that

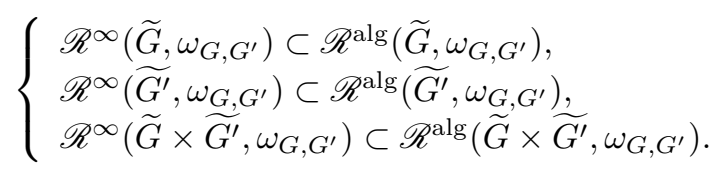

Then the algebraic version of the archimedean theta correspondence asserts that $\mathscr{R}^{\text {alg }}\left(\widetilde{G} \times \widetilde{G^{\prime}}, \omega_{G, G^{\prime}}\right)$ is the graph of a bijection (algebraic theta correspondence) between $\mathscr{R}^{\text {alg }}\left(\widetilde{G}, \omega_{G, G^{\prime}}\right)$ and $\mathscr{R}^{\text {alg }}\left(\widetilde{G^{\prime}}, \omega_{G, G^{\prime}}\right)$. In [Ho3, Roger Howe first proves this assertion, and then concludes the smooth version as a consequence of it. The following conjecture of the coincidence of the smooth version and the algebraic version of theta correspondence has been expected since the work of Howe.

Conjecture 1.2. The inclusions in (1.5) are all equalities.

Recall that the dual pair $\left(G, G^{\prime}\right)$ is said to be irreducible of type I if $A$ (or equivalently $A^{\prime}$ ) is a simple algebra, and it is said to be irreducible of type II if $A$ (or equivalently $A^{\prime}$ ) is the product of two simple algebras which are exchanged by $\tau$. Every dual pair is a direct product in an essentially unique way of irreducible dual pairs, and a complete classification of irreducible dual pairs has been given by Howe [Ho1].

Let $\mathbb{H}$ denote a quaternionic division algebra over $\mathbb{R}$, which is unique up to isomorphism. The following theorem is the main result of this paper.

Theorem 1.3. Conjecture 1.2 holds when the dual pair $\left(G, G^{\prime}\right)$ contains no quaternionic type I irreducible factor, that is, when A contains no $\tau$-stable ideal which is isomorphic to a matrix algebra $\mathrm{M}_{n}(\mathbb{H})(n \geq 1)$.

It is clear that it suffices to prove Theorem 1.3 for irreducible dual pairs. By the classification of irreducible dual pairs, we only need to show that Conjecture 1.2 
holds for (real or complex) orthogonal-symplectic dual pairs, unitary dual pairs, and all type II irreducible dual pairs. These are, respectively, proved in Sections 4. 5 and 3. For quaternionic type I irreducible dual pairs, we obtain a partial result in Proposition 3.2

\section{Some Cartan involutions}

Let $\left(G, G^{\prime}\right)$ be a reductive dual pair in $\operatorname{Sp}(W)$ as in the Introduction. We view both $G$ and $G^{\prime}$ as Nash groups. See $\mathrm{Su}$ ] for generalities on (almost linear) Nash groups. By a Cartan involution on $G$, we mean an involutive Nash automorphism whose fixed point set is a maximal compact subgroup of $G$. Note that the Cartan involution is determined by the associated maximal compact subgroup. Thus all Cartan involutions on $G$ are conjugate to each other by the adjoint action of $G$, since all maximal compact subgroups of $G$ are conjugate to each other. We remark that the "Nash" condition is necessary for the definition of a Cartan involution; for example, if $G=\mathbb{C}^{\times}$, then there are infinitely many involutive continuous automorphisms of $G$ whose fixed point set equals the maximal compact subgroup U(1) of $G$. The notion of Cartan involutions is similarly defined for arbitrary reductive Nash groups (see [Su, Section 15]).

Recall that a hyperbolic Nash group is a Nash group which is Nash isomorphic to $\left(\mathbb{R}_{+}^{\times}\right)^{n}$ for some $n \geq 0$, where $\mathbb{R}_{+}^{\times}$denotes the Nash group of all positive real numbers (see $[\mathrm{Su}$, Section 6$]$ ). Denote by $\Lambda$ the largest hyperbolic Nash subgroup in the center of $G$. For example, $\Lambda=\mathbb{R}_{+}^{\times}$if $G=\mathrm{GL}_{n}(\mathbb{R}), \mathrm{GL}_{n}(\mathbb{C})$ or $\mathrm{GL}_{n}(\mathbb{H})$ $(n \geq 1)$, and $\Lambda$ is trivial if $G$ is other classical groups. Note that $\Lambda$ also equals the largest hyperbolic Nash subgroup in the center of $G^{\prime}$.

As mentioned in the Introduction, the existence assertion of the following proposition is well known.

Proposition 2.1. Let $\sigma$ and $\sigma^{\prime}$ be Cartan involutions on $G$ and $G^{\prime}$, respectively. Then there exists a Cartan involution $\theta$ on $\operatorname{Sp}(W)$ extending both $\sigma$ and $\sigma^{\prime}$. Moreover, such a $\theta$ is unique up to conjugation by a unique element of $\Lambda$.

Proof. Note that every Cartan involution $\theta$ on $\operatorname{Sp}(W)$ is uniquely of the form $g \mapsto$ $J g J^{-1}$, where $J: W \rightarrow W$ is a $\mathbb{R}$-linear automorphism such that

- $J^{2}=-1$,

- $(u, v) \mapsto\langle J u, v\rangle_{W}$ is a positive definite symmetric bilinear form on $W$.

The condition that $\theta$ extends both $\sigma$ and $\sigma^{\prime}$ is equivalent to saying that

$$
J\left(g g^{\prime} . u\right)=\left(\sigma(g) \sigma\left(g^{\prime}\right)\right) . J u, \quad \text { for all } g \in G, g^{\prime} \in G^{\prime}, u \in W .
$$

Note that (2.1) implies that $J$ stabilizes every non-degenerate $G \times G^{\prime}$-subrepresentation of $W$. This reduces the proof to the case when the dual pair is irreducible. Then it is routine to verify the proposition case by case. We omit the details.

It is clear that Proposition 2.1 implies Proposition 1.1

\section{TYPE II IRREDUCIBLE DUAL PAIRS}

Let the notation be as in the Introduction. Recall that for each closed subgroup $E$ of $\operatorname{Sp}(W), \widetilde{E}$ denotes its double cover induced by the metaplectic cover $\widetilde{\operatorname{Sp}}(W) \rightarrow$ $\operatorname{Sp}(W)$. If $E$ is reductive as a Lie group, then so is $\widetilde{E}$, and we write $\operatorname{Irr}^{\operatorname{gen}}(\widetilde{E})$ for the subset of $\operatorname{Irr}(\widetilde{E})$ of genuine irreducible Casselman-Wallach representations. 
Here and as usual, "genuine" means that the representation does not descend to a representation of $E$.

Note that by the one-one correspondence property of local theta correspondence, if one of the three inclusions in (1.5) is an equality, then so are the other two. We first treat the simplest case of type II irreducible dual pairs.

Proposition 3.1. Conjecture 1.2 holds for all type II irreducible dual pairs.

Proof. Suppose that $\left(G, G^{\prime}\right)=\left(\mathrm{GL}_{r}(D), \mathrm{GL}_{s}(D)\right)$ is a type II irreducible dual pair, where $D=\mathbb{R}, \mathbb{C}$ or $\mathbb{H}$. Without loss of generality, assume that $r \leq s$. By GodementJacquet zeta integrals [GJ, Section I.8] and Kudla's persistence principle [Ku], we know that all representations in $\operatorname{Irr}^{\text {gen }}(\widetilde{G})$ occur in the smooth theta correspondence, namely,

$$
\operatorname{Irr}^{\operatorname{gen}}(\widetilde{G}) \subset \mathscr{R}^{\infty}\left(\widetilde{G}, \omega_{G, G^{\prime}}\right) .
$$

Since

$$
\mathscr{R}^{\infty}\left(\widetilde{G}, \omega_{G, G^{\prime}}\right) \subset \mathscr{R}^{\mathrm{alg}}\left(\widetilde{G}, \omega_{G, G^{\prime}}\right) \subset \operatorname{Irr}^{\mathrm{gen}}(\widetilde{G}),
$$

we conclude that $\mathscr{R}^{\infty}\left(\widetilde{G}, \omega_{G, G^{\prime}}\right)=\mathscr{R}^{\text {alg }}\left(\widetilde{G}, \omega_{G, G^{\prime}}\right)$. This proves the proposition.

Similar proof shows the following result for quaternionic dual pairs.

Proposition 3.2. Suppose that $\left(G, G^{\prime}\right)=\left(\operatorname{Sp}(p, q), \mathrm{O}^{*}(2 n)\right)$ is a quaternionic dual pair. If $p+q \leq n$, then the three inclusions in (1.5) are all equalities.

Proof. By [SZ, Theorem 7.3],

$$
\operatorname{Irr}^{\operatorname{gen}}(\widetilde{G}) \subset \mathscr{R}^{\infty}\left(\widetilde{G}, \omega_{G, G^{\prime}}\right) .
$$

Then the proposition follows by the same argument as in the proof of Proposition 3.1 .

\section{Orthogonal-SYMPleCtiC DUAL PAIRS}

We are aimed to prove the following proposition in this section.

Proposition 4.1. Conjecture 1.2 holds for all real orthogonal-symplectic dual pairs and all complex orthogonal-symplectic dual pairs.

In this section, let $\mathbb{F}$ be either the field $\mathbb{R}$ of real numbers, or the field $\mathbb{C}$ of complex numbers. Let $U$ be a finite-dimensional vector space over $\mathbb{F}$, equipped with a nondegenerate symmetric bilinear form on it. Let $G$ denote the isometry group of $U$, which is either a real orthogonal group or a complex orthogonal group. Fix a Cartan involution $\sigma$ on $G$. For each $n \geq 0$, we also fix an arbitrary Cartan involution $\sigma_{n}$ on the symplectic group $\operatorname{Sp}_{2 n}(\mathbb{F})$. Using Proposition 2.1, the pair $\left(\sigma, \sigma_{n}\right)$ determines a Cartan involution $\theta_{n}$ on the larger symplectic group $\operatorname{Sp}_{2 n \cdot \operatorname{dim}_{\mathbb{R}} U}(\mathbb{R})$. This yields a

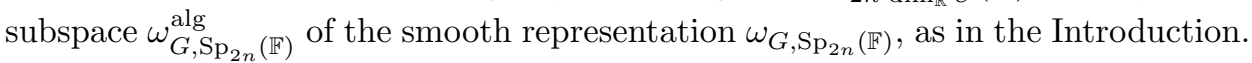

After twisting by an appropriate character, the representation of the double cover of $G$ on $\omega_{G, \mathrm{Sp}_{2 n}(\mathbb{F})}$ descends to a representation of $G$, which is realized on the Schwartz function space $\mathcal{S}\left(\mathbb{F}^{n \cdot \operatorname{dim}_{\mathbb{F}} U}\right)$ with the standard $G$-action. The reader is referred to $\left[\mathrm{Mo}\right.$ and $\mathrm{AB} 2$ for more details. The space $\omega_{G, \mathrm{Sp}_{2 n}(\mathbb{F})}^{\mathrm{alg}}$ corresponds to a subspace of $\mathcal{S}\left(\mathbb{F}^{n \cdot \operatorname{dim}_{F} U}\right)$, which we denote by $\mathcal{S}\left(\mathbb{F}^{n \cdot \operatorname{dim}_{\mathbb{F}} U}\right)^{\text {alg }}$.

Let $\pi \in \operatorname{Irr}(G)$. Define its first occurrence index

$$
\mathrm{n}(\pi):=\min \left\{n \geq 0 \mid \operatorname{Hom}_{G}\left(\mathcal{S}\left(\mathbb{F}^{n \cdot \operatorname{dim}_{\mathbb{F}} U}\right), \pi\right) \neq 0\right\} .
$$


Define the algebraic analogue

$$
\mathrm{n}^{\prime}(\pi):=\min \left\{n \geq 0 \mid \operatorname{Hom}_{G}^{\operatorname{alg}}\left(\mathcal{S}\left(\mathbb{F}^{n \cdot \operatorname{dim}_{\mathbb{F}} U}\right), \pi\right) \neq 0\right\},
$$

where $\operatorname{Hom}_{G}^{\text {alg }}$ is defined as in (1.4), by using the subspace of $\mathcal{S}\left(\mathbb{F}^{n \cdot \operatorname{dim}_{\mathbb{F}} U}\right)^{\text {alg }}$ of $\mathcal{S}\left(\mathbb{F}^{n \cdot \operatorname{dim}_{\mathbb{F}} U}\right)$. In view of Kudla's persistence principle $[\mathrm{Ku}$, it suffices to show that

$$
\mathrm{n}(\pi)=\mathrm{n}^{\prime}(\pi) \text {. }
$$

Let sgn denote the determinant character of $G$. The following lemma is a direct consequence of the correspondence in the joint harmonics (cf. Ho3]). An explicit computation of the correspondence in the joint harmonics can be found in [A Proposition 7.5]. The computation can be easily obtained by the duality correspondence for compact dual pairs (see [KV], EWH] and [Ho2]).

Lemma 4.2. The inequality

$$
\mathrm{n}^{\prime}(\operatorname{sgn}) \geq \operatorname{dim}_{\mathbb{F}} U
$$

holds.

Proof. See [Ho3, Lemma 4.1] and [A, Proposition 7.5].

By a well-know argument (cf. [Pr, Theorem 3.6.1 and Corollary C.43] and AB1, Proposition 1.7]), Lemma 4.2 implies the following lemma.

Lemma 4.3. The inequality

$$
\mathrm{n}^{\prime}(\pi)+\mathrm{n}^{\prime}(\pi \otimes \operatorname{sgn}) \geq \operatorname{dim}_{\mathbb{F}} U
$$

holds.

On the other hand, by the conservation relation ([SZ, Theorem 7.1]), we have that

$$
\mathrm{n}(\pi)+\mathrm{n}(\pi \otimes \operatorname{sgn})=\operatorname{dim}_{\mathbb{F}} U .
$$

Together with the obvious inequalities

$$
\mathrm{n}^{\prime}(\pi) \leq \mathrm{n}(\pi) \quad \text { and } \quad \mathrm{n}^{\prime}(\pi \otimes \operatorname{sgn}) \leq \mathrm{n}(\pi \otimes \operatorname{sgn}),
$$

(4.1) and (4.2) imply that $\mathrm{n}(\pi)=\mathrm{n}^{\prime}(\pi)$. This proves Proposition 4.1.

\section{UNITARY DUAL PAIRS}

For the proof of Theorem 1.3 it remains to show the following proposition.

Proposition 5.1. Conjecture 1.2 holds for all unitary dual pairs.

In this section, let $G$ be a unitary group attached to a complex skew-Hermitian space of dimension $n \geq 0$. Let $\delta \in \mathbb{Z} / 2 \mathbb{Z}$. Define

$$
G_{\delta}:= \begin{cases}G \times\{1,-1\}, & \text { if } \delta=0 \\ \left\{(g, t) \in G \times \mathbb{C}^{\times} \mid \operatorname{det} g=t^{2}\right\}, & \text { if } \delta=1 .\end{cases}
$$

This is a double cover of $G$. Note that the double cover of $G$ associated to the dual pair $(G, \mathrm{U}(p, q))$ is canonically isomorphic to $G_{\delta}$, where $\mathrm{U}(p, q)$ is the unitary group attached to the complex Hermitian space of signature $(p, q)(p, q \geq 0)$, with $p-q$ having parity $\delta$. See [Pa1, Section 1] for details.

As in the last section, fix a Cartan involution on $G$, and for all $p, q \geq 0$, fix a Cartan involution of $\mathrm{U}(p, q)$. Using Proposition 2.1 this determines a Cartan 
involution on the larger symplectic group $\operatorname{Sp}_{2 n(p+q)}(\mathbb{R})$, which further determines a subspace $\omega_{G, \mathrm{U}(p, q)}^{\mathrm{alg}}$ of the smooth representation $\omega_{G, \mathrm{U}(p, q)}$.

Let $\pi \in \operatorname{Irr}^{\mathrm{gen}}\left(G_{\delta}\right)$. For every integer $t$ with parity $\delta$, define the first occurrence index

$$
\mathrm{n}_{t}(\pi):=\min \left\{p+q \mid p, q \geq 0, p-q=t, \operatorname{Hom}_{G_{\delta}}\left(\omega_{G, \mathrm{U}(p, q)}, \pi\right) \neq 0\right\} .
$$

Define its algebraic analogue

$$
\mathrm{n}_{t}^{\prime}(\pi):=\min \left\{p+q \mid p, q \geq 0, p-q=t, \operatorname{Hom}_{G_{\delta}}^{\mathrm{alg}}\left(\omega_{G, \mathrm{U}(p, q)}, \pi\right) \neq 0\right\},
$$

where $\operatorname{Hom}_{G_{\delta}}^{\mathrm{alg}}$ is defined as in (1.4).

Recall the following fact of conservation relations from [SZ, Theorem 7.6].

Lemma 5.2. There are two distinct integers $t_{1}$ and $t_{2}$, both of parity $\delta$, such that

$$
\mathrm{n}_{t_{1}}(\pi)+\mathrm{n}_{t_{2}}(\pi)=2 n+2 .
$$

In the algebraic setting, we need the following lemma.

Lemma 5.3. Assume that $\delta=0$ and let $t$ be a non-zero even integer. Then

$$
\mathrm{n}_{t}^{\prime}\left(1_{U}\right)=2 n+|t|
$$

where $1_{U} \in \operatorname{Irr}^{\mathrm{gen}}\left(G_{\delta}\right)$ denotes the representation with trivial action of $G \subset G_{\delta}$.

Proof. See [Pa2, Lemma 3.1].

As in the last section, Lemma 5.3 implies the following lemma (cf. the proof of [SZ, Theorem 7.6]).

Lemma 5.4. Let $t_{1}$ and $t_{2}$ be two distinct integers, both of parity $\delta$. Then

$$
\mathrm{n}_{t_{1}}^{\prime}(\pi)+\mathrm{n}_{t_{2}}^{\prime}(\pi) \geq 2 n+\left|t_{1}-t_{2}\right| .
$$

We need the following lemma.

Lemma 5.5. Let $t$ be an integer with parity $\delta$. If $\mathrm{n}_{t}^{\prime}(\pi) \leq n$, then $\mathrm{n}_{t}(\pi)=\mathrm{n}_{t}^{\prime}(\pi)$.

Proof. Let $t_{1}$ and $t_{2}$ be as in Lemma 5.2. Together with the obvious inequalities

$$
\mathrm{n}_{t_{1}}^{\prime}(\pi) \leq \mathrm{n}_{\mathrm{t}_{1}}(\pi) \quad \text { and } \quad \mathrm{n}_{t_{2}}^{\prime}(\pi) \leq \mathrm{n}_{t_{2}}(\pi),
$$

(5.1) and (5.2) imply that

$$
\mathrm{n}_{t_{1}}^{\prime}(\pi)=\mathrm{n}_{\mathrm{t}_{1}}(\pi) \quad \text { and } \quad \mathrm{n}_{t_{2}}^{\prime}(\pi)=\mathrm{n}_{t_{2}}(\pi) .
$$

Thus it suffices to show that $t=t_{1}$ or $t_{2}$. Assume this is not true. Then Lemma 5.4 implies that

$$
\mathrm{n}_{t}^{\prime}(\pi)+\mathrm{n}_{t_{i}}^{\prime}(\pi) \geq 2 n+2, \quad i=1,2 .
$$

Together with the inequality $\mathrm{n}_{t}^{\prime}(\pi) \leq n$, (5.4) implies that

$$
\mathrm{n}_{t_{1}}^{\prime}(\pi)+\mathrm{n}_{t_{2}}^{\prime}(\pi) \geq 2 n+4
$$

This contradicts (5.1) and (5.3). 
Finally, we come to the proof of Proposition 5.1 for the dual pair $(G, \mathrm{U}(p, q))$. Without loss of generality, assume that $p+q \leq n$. Suppose that $p+q$ has parity $\delta$ and $\pi \in \mathscr{R}^{\mathrm{alg}}\left(G_{\delta}, \omega_{G, \mathrm{U}(p, q)}\right)$. Then

$$
\mathrm{n}_{p-q}^{\prime}(\pi) \leq p+q \leq n
$$

Thus by Lemma 5.5

$$
\mathrm{n}_{p-q}(\pi)=\mathrm{n}_{p-q}^{\prime}(\pi) \leq p+q .
$$

This implies that $\pi \in \mathscr{R}^{\infty}\left(G_{\delta}, \omega_{G, \mathrm{U}(p, q)}\right)$, and Proposition 5.1 is proved.

\section{ACKNOWLEDGMENT}

The authors are very grateful to the referee for the insightful comments.

\section{REFERENCES}

[A] J. Adams, The theta correspondence over $\mathbb{R}$, Harmonic analysis, group representations, automorphic forms and invariant theory, Lect. Notes Ser. Inst. Math. Sci. Natl. Univ. Singap., vol. 12, World Sci. Publ., Hackensack, NJ, 2007, pp. 1-39, DOI 10.1142/9789812770790_0001. MR2401808

[AB1] J. Adams and D. Barbasch, Reductive dual pair correspondence for complex groups, J. Funct. Anal. 132 (1995), no. 1, 1-42, DOI 10.1006/jfan.1995.1099. MR.1346217

[AB2] J. Adams and D. Barbasch, Genuine representations of the metaplectic group, Compositio Math. 113 (1998), no. 1, 23-66, DOI 10.1023/A:1000450504919. MR1638210

[BK] J. Bernstein and B. Krötz, Smooth Fréchet globalizations of Harish-Chandra modules, Israel J. Math. 199 (2014), no. 1, 45-111, DOI 10.1007/s11856-013-0056-1. MR3219530

[Ca] W. Casselman, Canonical extensions of Harish-Chandra modules to representations of $G$, Canad. J. Math. 41 (1989), no. 3, 385-438, DOI 10.4153/CJM-1989-019-5. MR1013462

[EWH] T. Enright, R. Howe, and N. Wallach, A classification of unitary highest weight modules, Representation theory of reductive groups (Park City, Utah, 1982), Progr. Math., vol. 40, Birkhäuser Boston, Boston, MA, 1983, pp. 97-143. MR733809

[GJ] R. Godement and H. Jacquet, Zeta functions of simple algebras, Lecture Notes in Mathematics, Vol. 260, Springer-Verlag, Berlin-New York, 1972. MR0342495

[Ho1] R. Howe, $\theta$-series and invariant theory, Automorphic forms, representations and $L$ functions (Proc. Sympos. Pure Math., Oregon State Univ., Corvallis, Ore., 1977), Proc. Sympos. Pure Math., XXXIII, Amer. Math. Soc., Providence, R.I., 1979, pp. 275-285. MR546602

[Ho2] R. Howe, Remarks on classical invariant theory, Trans. Amer. Math. Soc. 313 (1989), no. 2, 539-570, DOI 10.2307/2001418. MR986027

[Ho3] R. Howe, Transcending classical invariant theory, J. Amer. Math. Soc. 2 (1989), no. 3, 535-552, DOI 10.2307/1990942. MR.985172

[KV] M. Kashiwara and M. Vergne, On the Segal-Shale-Weil representations and harmonic polynomials, Invent. Math. 44 (1978), no. 1, 1-47, DOI 10.1007/BF01389900. MR0463359

$[\mathrm{Ku}] \quad$ S. S. Kudla, On the local theta-correspondence, Invent. Math. 83 (1986), no. 2, 229-255, DOI 10.1007/BF01388961. MR818351

[Mo] C. Mœglin, Correspondance de Howe pour les paires reductives duales: quelques calculs dans le cas archimédien (French), J. Funct. Anal. 85 (1989), no. 1, 1-85, DOI 10.1016/0022-1236(89)90046-3. MR1005856

[MW] C. Mœglin, M.-F. Vignéras, and J.-L. Waldspurger, Correspondances de Howe sur un corps p-adique (French), Lecture Notes in Mathematics, vol. 1291, Springer-Verlag, Berlin, 1987. MR 1041060

[Pa1] A. Paul, Howe correspondence for real unitary groups, J. Funct. Anal. 159 (1998), no. 2, 384-431, DOI 10.1006/jfan.1998.3330. MR.1658091

$[\mathrm{Pa} 2]$ A. Paul, First occurrence for the dual pairs $(\mathrm{U}(p, q), \mathrm{U}(r, s))$, Canad. J. Math. 51 (1999), no. 3, 636-657, DOI 10.4153/CJM-1999-029-6. MR1701329

[Pr] T. Przebinda, The oscillator duality correspondence for the pair $\mathrm{O}(2,2), \mathrm{Sp}(2, \mathbf{R})$, Mem. Amer. Math. Soc. 79 (1989), no. 403, x+105, DOI 10.1090/memo/0403. MR979944 
[Rao] R. Ranga Rao, On some explicit formulas in the theory of Weil representation, Pacific J. Math. 157 (1993), no. 2, 335-371. MR1197062

[Sh] D. Shale, Linear symmetries of free boson fields, Trans. Amer. Math. Soc. 103 (1962), 149-167, DOI 10.2307/1993745. MR0137504

[Su] B. Sun, Almost linear Nash groups, Chin. Ann. Math. Ser. B 36 (2015), no. 3, 355-400, DOI 10.1007/s11401-015-0915-7. MR3341164

[SZ] B. Sun and C.-B. Zhu, Conservation relations for local theta correspondence, J. Amer. Math. Soc. 28 (2015), no. 4, 939-983, DOI 10.1090/S0894-0347-2014-00817-1. MR3369906

[Wa] N. R. Wallach, Real reductive groups. II, Pure and Applied Mathematics, vol. 132, Academic Press, Inc., Boston, MA, 1992. MR.1170566

[We] A. Weil, Sur certains groupes d'opérateurs unitaires (French), Acta Math. 111 (1964), 143-211, DOI 10.1007/BF02391012. MR0165033

School of Sciences, Harbin Institute of Technology, Shenzhen, 518055, China

E-mail address: baoyixin@hit.edu.cn

Academy of Mathematics and Systems Science, Chinese Academy of Sciences - AndSchool of Mathematics, University of Chinese Academy of Sciences, Beijing, 100190, China

E-mail address: sun@math.ac.cn 